\title{
Thermohydraulique de la zone non saturée en relation avec les échanges sol-atmosphère
}

\author{
Thermohydraulics in the unsaturated zone \\ in relation with soil-atmosphere exchanges
}

\author{
Y. Brunet \\ Institut national de la \\ recherche agronomique \\ Station de bioclimatologie \\ Montfavet
}

\author{
M. Vauclin, G. Vachaud \\ Institut de mécanique de Grenoble \\ Saint-Martin d'Hères
}

\begin{abstract}
On présente d'abord une modélisation conceptuelle des transferts hydriques et thermiques avec changement de phase dans une tranche verticale de sol non saturé, couplés aux échanges de masse, de chaleur et de quantité de mouvement avec l'atmosphère.

A partir d'expériences numériques, l'accent est ensuite mis sur les points suivants : sensibilité du comportement de la zone superficielle du sol aux variables microclimatiques et aux paramètres de surface et du sol; influence de la variabilité spatiale du sol sur les échanges avec l'atmosphère (une modélisation stochastique des transferts dans le sol est proposée); paramétrisation de l'évolution temporelle de la température de surface, compatible avec les contraintes imposées par une modélisation sur de longues périodes.
\end{abstract}

First is described a conceptual model of hydric and thermal transfers with change of phase in a vertical segment of unsaturated soil, coupled with mass, heat and quantity of movement exchanges with the atmosphere.

From numerical experiments, the following points are then stressed: sensitivity of the behavior of the soil surface to microclimatic variations and surface and soil parameters: influence of the variability in space of the soil on exchanges with the atmosphere (a stochastic model of transfers in the soil is proposed); parameterizing of the variation with time of the surface temperature, compatible with the constraints imposed by modeling over long periods.

\section{Introduction}

Une approche fine du problème thermique dans le sous-sol nécessite une identification et une modélisation correctes des phénomènes en jeu : processus de transferts et mécanismes de couplage, mais aussi conditions aux limites, qui vont en régler l'évolution et l'intensité.

En matière de transfert thermique dans les sols, la méthode la plus simple consiste à traiter un problème exclusivement conductif dans une tranche de sol encadrée par deux niveaux de mesure ou d'estimation de températures et/ou de flux.

Cette approche se heurte dans la majorité des cas au poids des hypothèses alors mises en jeu :

- au niveau des conditions aux limites, parce que dans une optique de simulation en conditions naturelles, de prévision, d'étude de gestion du gisement solaire, il est tout à fait opportun de pouvoir calculer les champs thermiques à partir des données climatiques au voisinage de la surface du sol, véritables "fonctions de forçage "; - au niveau du modèle de transfert lui-même, puisque :

- les transferts thermiques ne sont pas purement conductifs (transport de chaleur latente, thermo-migration de l'eau liquide);

- la forte dépendance des coefficients de transfert thermique vis-à-vis des conditions hydriques du milieu poreux considéré rend nécessaire le calcul du champ de pression;

- le calcul de la température ou du flux de chaleur à la surface du sol à partir des données climatiques met en jeu la pression d'eau à cette surface et impose donc lui aussi la résolution du problème hydrique. 
Ces réflexions nous amèneront d'abord à décrire succinctement les mécanismes d'échange de chaleur, de masse et de quantité de mouvement entre sol et atmosphère, puis à proposer un modèle mécaniste de transferts hydrique et thermique dans une tranche verticale de sol non saturé.
Nous montrerons alors que le couplage de ces deux ensembles de lois permet le calcul des champs de température et d'humidité à partir des variables climatiques classiques à un niveau de référence : rayonnement, vitesse du vent, température et pression de vapeur de l'air.

\section{Modélisation locale \\ des transferts thermiques et hydriques dans le système sol-atmosphère}

\subsection{Description des échanges sol-atmosphère}

La source première d'énergie à la surface du sol est de nature radiative. Celle-ci reçoit en effet des rayonnements d'origine solaire (le rayonnement global $R g$ ) et atmosphérique $(R a)$, et émet un rayonnement propre; le bilan de ces échanges est le rayonnement net $R n$, qui peut s'écrire :

$$
R n=(1-a) R g+\varepsilon R a-\sigma \varepsilon T s^{4}
$$

L'albédo $a$ et l'émissivité $\varepsilon$ sont caractéristiques de la surface, dont Ts est la température; $\sigma$ est la constante de Stefan-Boltzmann.

Ce rayonnement net, positif le jour et négatif la nuit, se convertit à la surface du sol en flux conductif $S$ et en flux convectifs : flux de chaleur sensible $H$ et flux de chaleur latente $L E$ associé au flux de masse d'eau $E$ qui se vaporise - ou se condense - à la surface. Le flux de chaleur entrant dans le sol est donc le résultat du bilan de ces différentes formes d'énergie

$$
S=R n-H-L E
$$

( $S, H$ et $L E$ étant positifs lorsqu'ils quittent la surface).

Les flux convectifs peuvent s'exprimer à partir des équations de la mécanique des fluides appliquées à l'écoulement atmosphérique, et compte tenu d'un certain nombre d'hypothèses: homogénéité et stationnarité de la couche limite notamment. On montre alors que les profils de vitesse du vent, de température et d'humidité spécifique peuvent dans le cas général de la convection mixte, s'écrire de la façon suivante, pour $z<<\max \left(z_{o m}, z_{o h}, z_{o v}\right)$ :

$$
\begin{gathered}
\frac{\bar{u}}{u \cdot}=\frac{1}{k}\left(\log \frac{z}{z_{o m}}-\psi_{m}\left(\frac{z}{L}\right)\right) \\
\frac{\bar{\theta}-\bar{\theta}_{s}}{\theta}=\frac{1}{k}\left(\log \frac{z}{z_{o h}}-\psi_{h}\left(\frac{z}{L}\right)\right) \\
\frac{\bar{q}-\bar{q}_{s}}{q \cdot}=\frac{1}{k}\left(\log \frac{z}{z_{o v}}-\psi_{v}\left(\frac{z}{L}\right)\right)
\end{gathered}
$$

Dans ces équations, $\bar{u}, \bar{\theta}$ et $\bar{q}$ sont respectivement les vitesse du vent, température potentielle et humidité spécifique mesurées à l'altitude $z ; z_{o m}, z_{o h}$ et $z_{o v}$ sont les " hauteurs de rugosité ", constantes d'intégration des différents profils, auxquelles les grandeurs correspondan- tes prennent leur valeur de surface $\bar{U}_{s}=0, \theta$, et $\bar{q}_{s} ; k$ est la constante de Von Karman ( $k=0.4)$, et $L$ la longueur de Monin-Obukhov, caractéristique de l'importance des forces de flottabilité, et définie par :

$$
L=-\frac{\rho C_{p} \theta u^{3}}{k g H}
$$

$u \cdot, \theta \cdot, q$ sont des échelles de vitesse, température et humidité

$$
u_{*}=\sqrt{\frac{\tau_{0}}{\rho}} ; \theta=-\frac{H}{\rho C_{p} u_{*}} ; q \cdot=-\frac{E}{\rho_{l} u_{v}}
$$

( $\tau_{0}$ : contrainte pariétale ou flux de quantité de mouvement); enfin, les fonctions $\psi_{m}, \psi_{h}$ et $\psi_{v}$ sont des fonctions universelles, dont les valeurs ont été déterminées expérimentalement (voir par exemple BRUTSAERT, 1982, pour une revue d'ensemble).

De la sorte, les flux convectifs peuvent s'exprimer comme proportionnels à l'écart des variables d'état correspondantes entre la surface et le niveau de mesure :

$$
\begin{aligned}
H & =\rho C_{p} h_{h}\left(\bar{\theta}_{s}-\bar{\theta}\right) \\
L E & =\rho_{l} L_{v} h_{v}\left(\bar{q}_{s}-\bar{q}\right)
\end{aligned}
$$

avec :

$$
\begin{aligned}
& h_{h}=u \cdot \theta \cdot /\left(\bar{\theta}-\bar{\theta}_{s}\right) \\
& h_{v}=u \cdot q \cdot /\left(\bar{q}-\bar{q}_{s}\right)
\end{aligned}
$$

Ces équations ne sont valables que si $z$ est inférieure à une hauteur $z_{h}$ caractéristique du site considéré (rugosité de surface, distance au bord d'attaque, caractéristiques de la zone amont...): typiquement, $z_{h}=1 \mathrm{~m}$ pour une distance minimale au bord d'attaque de l'ordre de 10 à $100 \mathrm{~m}$, suivant les conditions.

Le bilan d'énergie à la surface du sol fait donc intervenir :

- les variables de forçage atmosphérique : $R g, \bar{u}, \bar{\theta}, \bar{q}$ (le rayonnement atmosphérique $R a$ pouvant s'exprimer à partir de $\theta$ et $\bar{q}$; BRUTSAERT, 1975);

- des paramètres, dépendant de la surface elle-même : $a, \varepsilon, z_{o m}$;

- des variables d'équilibre : $\theta_{s}$ et $\tilde{q}_{s}$.

Le flux de chaleur dans le sol peut s'exprimer lui, d'une manière que nous allons maintenant envisager. 


\subsection{Description locale des transferts thermo-hydrauli- ques dans le sol}

Les transferts thermiques et hydriques en milieu poreux peuvent être modélisés de manière relativement simple et classique (voir dans le même numéro les articles de BoRIEs, et Benet, JouanNa), si l'on suppose valides un certain nombre d'hypothèses: introduction de la notion de " volume élémentaire représentatif ", homogénéité et indéformabilité du milieu, absence de réaction chimique, accélération et vitesse d'écoulement faibles, transferts de chaleur par rayonnement négligeables, pression totale de la phase gazeuse uniforme.

Leur formalisme s'établit à partir d'équations locales de conservation (masse, quantité de mouvement, énergie), d'équations d'état et d'équilibre, de lois constitutives dont l'utilisation nécessite la connaissance de coefficients phénoménologiques.

L'ensemble d'équations utilisé ici (BRUNET, 1984), valable lorsque la température de la phase liquide ne lui permet ni de se congeler, ni de s'autovaporiser, est résumé dans le tableau I. Il apparait clairement que la conduction thermique pure dans le sol n'est qu'un aspect particulier d'un ensemble complexe de processus couplés que rien a priori n'autorise à traiter séparément.

Pour les transferts de chaleur et de masse, les variables d'état retenues sont, respectivement, la température $T$ et la pression effective de l'eau $\mathrm{h}$; cette dernière est préférée à l'humidité volumique puisque son utilisation permet de simuler le comportement de la zone saturée comme celui de la zone non saturée, et de prendre en compte une éventuelle hétérogénéité du milieu poreux (VAUCLIN et al., 1979; Milly, 1982).

Dans la suite de l'exposé, nous nous limiterons au cas de transferts monodimensionnels verticaux (homogénéité horizontale du milieu).

\subsection{Le couplage sol-atmosphère}

Les deux ensembles de lois que nous venons d'envisager et qui se rapportent, respectivement, aux transferts dans l'atmosphère et le sol, peuvent être couplés en imposant à la surface du sol des contraintes de continuité :

- continuité des variables d'état

- égalité des températures potentielles et réelles

$$
\bar{\theta}_{s}=\bar{T}_{s}
$$

- continuité de l'humidité spécifique

$$
q_{s}=\frac{P_{V S}\left(T_{s}\right)}{\rho R_{v} T_{s}} \exp \left(\frac{g h_{s}}{R_{v} T_{s}}\right)
$$

- continuité des flux, qui boucle le système

- bilan d'énergie

$$
\begin{aligned}
R n-H-L E=-D_{c h} & \left.\frac{\partial h}{\partial z}\right|_{z=0}-\left.D_{c t} \frac{\partial T}{\partial z}\right|_{z=0} \\
& +\left.C_{l}\left(T-T_{0}\right) J_{m}\right|_{z=0}
\end{aligned}
$$

Tableau 1

Modélisation des transferts thermo-hydrauliques dans le sol. (La signification des symboles suit les recommandations de la $7^{\circ}$ Conférence Internationale sur les transferts de chaleur. Munich 1982).

Equations de conservation

$$
\begin{aligned}
& c_{h} \frac{\partial h}{\partial t}=\nabla \cdot\left(D_{m h} \nabla h+D_{m+} \nabla T\right)-\frac{\partial k \ell h}{\partial z} \\
& c_{T} \frac{\partial T}{\partial z}=\nabla \cdot\left(D_{c h} \nabla h+D_{c T} \nabla T\right)-C_{j} J_{m} \nabla T \\
& \text { Equations de flux } \\
& J_{1} / e_{1}=-k_{2} \nabla h-D_{2 T} \nabla T+K_{l h} i \\
& J_{v} / e_{l}=-D_{v h} \text { oh }-D_{v T} \pi T \\
& J_{m} / l_{l}=-D_{m h} \nabla h-D_{m T} \nabla T+K_{\text {Sh }} i \\
& J_{c} J_{c}=-D_{c h} \nabla h-D_{c T} \nabla T+C_{1}\left(T_{-} T_{0}\right) J_{m}
\end{aligned}
$$

Equations d'état et d'équilibre $p_{v} / e_{v}=R_{v} T ; p_{a} / e_{a}=R_{a} T$ $e_{r}=e_{r s}(T) \exp (g h / R, T) ; \rho_{l}=c^{\text {she }}$

$$
\eta=\eta(h, T)
$$

$$
\frac{\text { Coefficients de transport }}{K_{l h}=K_{l h}(h, T)}
$$$$
D_{\ell T}=-L_{Q T}\left(H_{R T}\right) / T \rho_{P}
$$$$
D_{v b}=\frac{\Lambda}{e_{t}} \nu_{0} \frac{P_{a}}{p_{a}-p_{v}}\left\{\left(\eta_{q}\right) \xi \frac{\partial e_{v}}{\partial h}\right.
$$$$
D_{v \tau}=\frac{1}{e_{l}} \nu_{v} \frac{P_{a}}{P_{a}-\rho_{r}} f\left(\eta_{t}\right) f^{\prime}\left(\eta_{g}\right) \times \frac{\partial e_{v}}{\partial T}
$$$$
D_{m h}=K_{\text {lh }}+D_{v h}
$$$$
D_{m} \tau=D_{\ell T}+D_{r}
$$$$
D_{C h}=g T D_{k T}+L_{V} D_{V h}
$$$$
D_{C T}=\lambda+e_{Y} L_{V} D_{V T}
$$

(1 : liquide, $v$ : vapeur, $m$ : masse, $c$ : chaleur, $g$ : air)

\section{Tableau 2}

\section{Caractéristiques du modèle}

Variables d'état

Paramètres

Variables de sortie

Fonctions de forcage

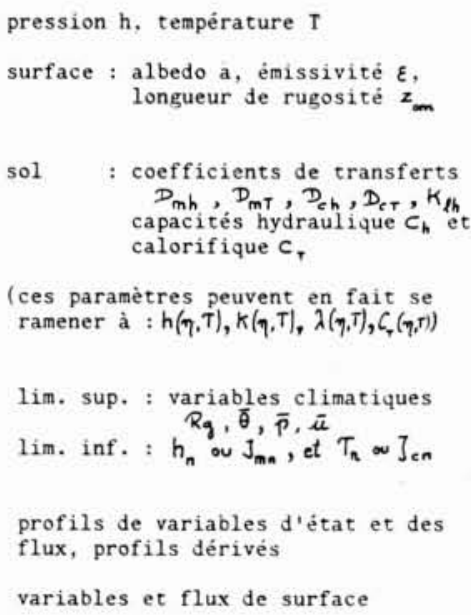

lim. sup. : variables climatiques lim. inf.: $h_{n}{ }_{\text {ou }}, \bar{J}, \bar{p}, \bar{\mu}$, et $\tau_{n}$ or $J_{\text {en }}$

profils de variables d'état et des flux, profils dérivés

variables et flux de surface 
- bilan de masse

$$
E-P=\left(\left.D_{\mathrm{mh}} \frac{\partial h}{\partial z}\right|_{z=0}+\left.D_{m T} \frac{\partial T}{\partial z}\right|_{z=0}-K_{l h}\right) \rho_{l}
$$

Ces contraintes fournissent les conditions à la limite supérieure du système-sol (première ou deuxième espèce). A la limite inférieure, on pourra prendre en compte un niveau de mesure, ou imposer la présence d'une nappe, d'un sous-sol imperméable, d'une zone à teneur en eau constante... Les conditions initiales sont déterminées par la connaissance des profils initiaux de température et pression (ou humidité).

Le problème mathématique à résoudre se présente donc sous la forme d'un système de deux équations aux dérivées partielles, paraboliques, d'ordre 2 , fortement non linéaires, assorti de ses conditions initiales et aux limites; les bilans de masse et d'énergie à la surface du sol formant un ensemble de deux équations non linéaires à deux inconnues ( $T_{s}$ et $h_{s}$ ). L'ensemble peut être résolu à l'aide d'un schéma numérique (éléments finis, différences finies ici) et d'un algorithme d'ajustement non linéaire.

Les caractéristiques du modèle sont résumées dans le tableau II.

On peut trouver des exemples de simulation réalisées avec des modèles de ce type dans Rosema (1975), VAN Bavel et Hillel (1976), VAuclin et al. (1977), Brunet (1982), RECAN (1982), BRUNET (1984).

L'un d'eux (BRUNET, 1984) a pu être validé, par comparaison avec des profils de température et d'humidité dans le sol.

\section{Analyse critique de l'approche et simplifications possibles}

Pris dans son intégralité, le modèle s'avère d'un emploi relativement lourd, et ce pour plusieurs raisons :

- ses équations comportent de nombreux termes, dans certains desquels figurent des coefficients mal connus (diffusion de la vapeur d'eau par exemple);

- certains paramètres du modèle, et notamment les coefficients de transfert dans le sol, sont particulièrement délicats à obtenir et nécessitent de nombreuses mesures qu'il est difficile de faire en routine;

- ces paramètres étant spatialement très variables, la forte non-linéarité des équations est a priori incompatible avec une utilisation non strictement locale du modèle;

- les schémas de résolution numérique imposent un pas de temps de calcul très faible (la figure 1 montre l'évolution du pas de temps au cours d'une journée de simulation, sous la contrainte que la variation relative de la conductivité hydraulique, d'une étape à la suivante, ne dépasse pas $10 \%$ ) : le coût peut devenir important, et les données d'entrée (variables atmosphériques) doivent être acquises à une fréquence suffisamment élevée.

Cette lourdeur pouvant s'avérer peu compatible avec un objectif de simulation du comportement thermique du sous-sol, il apparait tout à fait opportun de chercher à simplifier cette version de base. Dans cet esprit, nous allons envisager trois volets :

- étude du poids respectif des différents termes des équations et analyse de sensibilité aux paramètres du modèle;

- étude de l'influence de la variabilité spatiale des caractéristiques hydrodynamiques, et des moyens d'y faire face de manière simple;

- présentation d'une possible "paramétrisation " des transferts dans le sol, plus compatible avec une utilisation sur de plus grandes échelles de temps et d'espace.

\subsection{Poids des différents termes et analyse de sensibilité}

En ce qui concerne l'importance relative des divers termes des équations de transfert dans le sol, il est possible de dégager les points suivants :

- le rapport $c_{l} J_{m} \nabla T / \nabla\left(D_{c h} \nabla h+D_{c T} \nabla T\right)$ entre les termes convectifs et conductifs de l'équation de la chaleur n'atteint jamais $10 \%$, même pour des sols fortement perméables (DUNAND et al., 1980; RECAN, 1982) : l'absence du terme convectif ne modifie pas la température de plus de $0.1^{\circ} \mathrm{C}$;

- de rares études tendraient à montrer que l'influence du terme de mouillage n'est pas toujours négligeable, ni dans l'équation d'énergie (ANDERSON et LINVILLE, 1962), ni dans l'équation de masse (Groenevelt et KAY, 1974), et ce, en particulier dans le cas de matériaux argileux; le calcul du coefficient de transfert correspondant apparaît en outre assez complexe;

- si les transferts en phase vapeur semblent tout à fait négligeables devant les transferts liquides lorsque le milieu poreux présente une forte teneur en eau, il n'en va plus de même dès lors que les conditions climatiques provoquent un assèchement de la zone superficielle : le flux vapeur peut y devenir prédominant (CRAUSSE et al., 1981; RECAN, 1982; BRUNET, 1984). Ne pas en tenir compte pourrait modifier sensiblement le régime thermique, d'autant plus que le transport de chaleur latente peut alors représenter jusqu'à $30 \%$ du transfert de chaleur par conduction.

Un certain nombre d'analyses de sensibilité aux paramètres d'entrée du modèle ont été présentées dans la littérature, de manières assez diverses qui rendent malheureusement difficile une bonne synthèse. Quelques faits semblent cependant émerger nettement : 


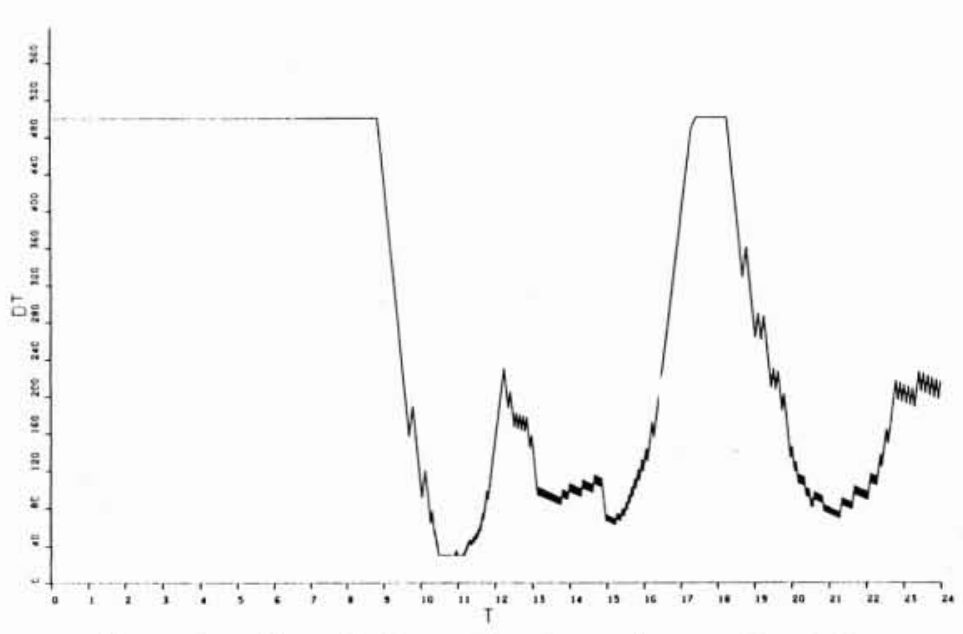

Figure 1. - Exemple de variation du pas de temps de calcul au cours d'une journée de simulation (d'après Brunet, 1984).

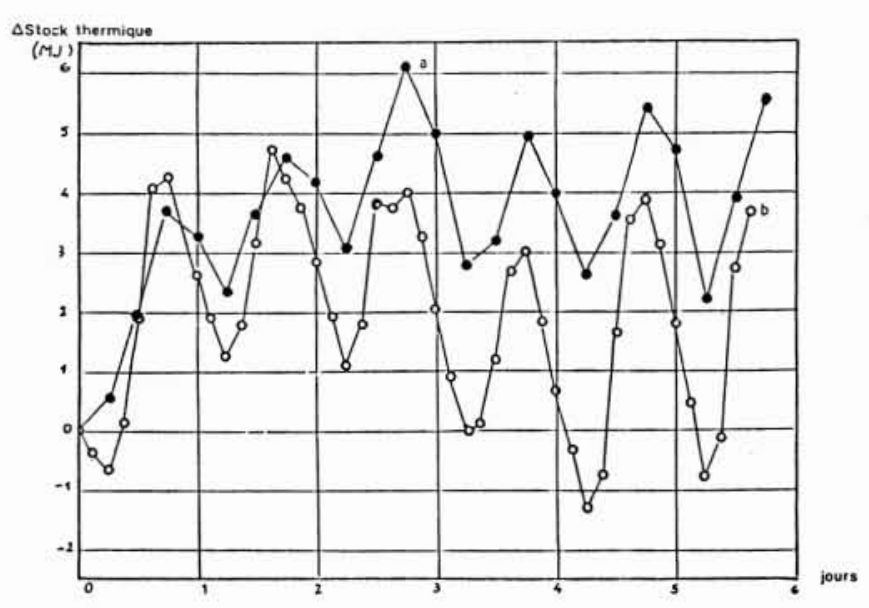

Figure 2. - Evolution des variations de stock thermique par rapport à l'instant initial (d'après Dunand et al., 1980)

- a - température de surface du sol = température de l'air.

○ b - température de surface du sol calculée par le bilan radiatif.

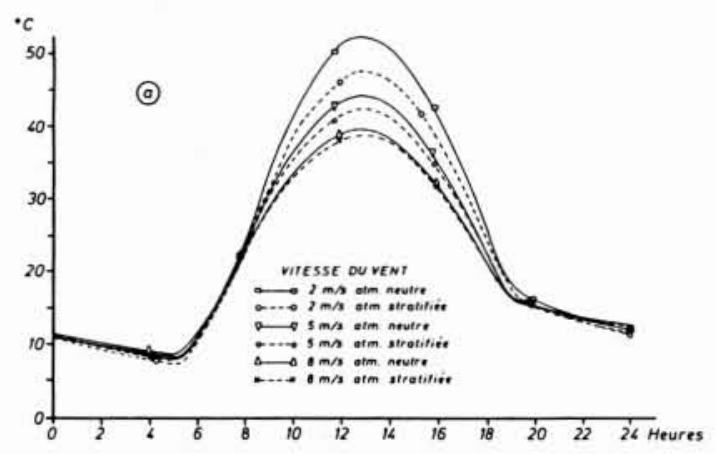

- le modèle se montre très sensible aux variables climatiques, en particulier rayonnement et température (ROSEMA, 1975; VAuCLin et al., 1977; BRUNET, 1982; RECAN, 1982); le fait, couramment utilisé, d'imposer à la surface du sol la température de l'air peut provoquer des erreurs notables (fig. 2);

- une variation relative d'albedo ou d'émissivité de $20 \%$ modifie la température de surface de 0.2 à $2{ }^{\circ} \mathrm{C}$ suivant les cas (Rosema, 1975; VAUCLIN et al., 1977);

- le modèle n'est pas très sensible à la longueur de rugosité aérodynamique, pour peu que l'on adopte une formulation correcte des longueurs de rugosité thermique et massique (BRUNET, 1982; RECAN, 1982; fig. $3 b$ et c); il s'avère par contre plus sensible, aux faibles vitesses du vent, à la prise en compte de la stratification thermique (influence du paramètre $\mathrm{z} / \mathrm{L}$, fig. $3 a$ )
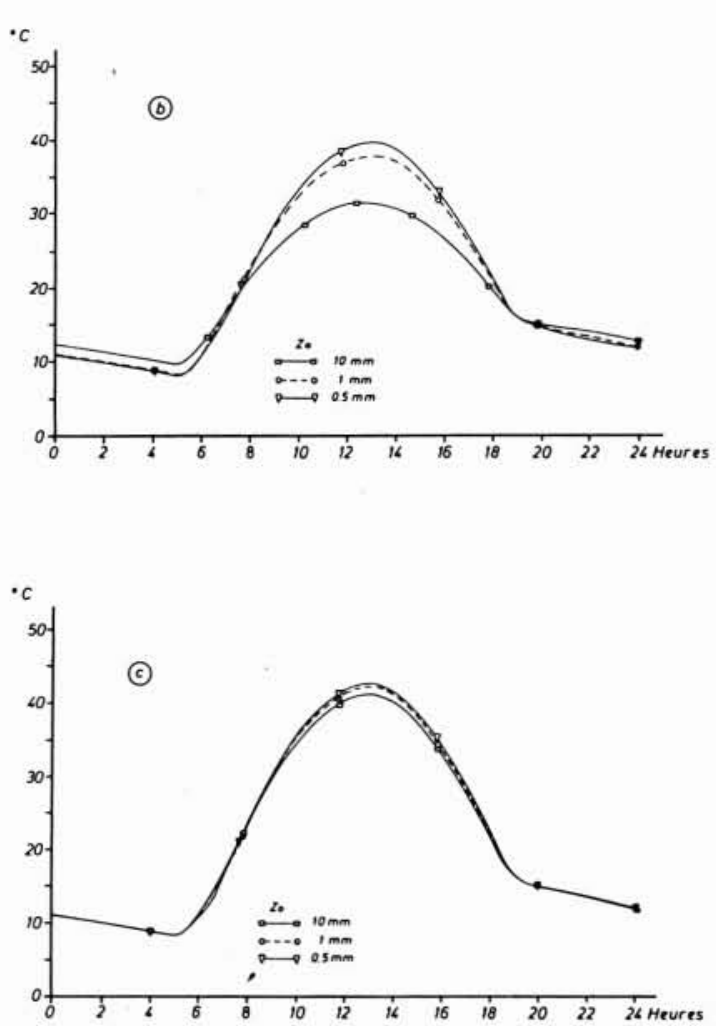

Figure 3. - Influence de divers paramètres sur le calcul de la température de surface (d’après Récan 1982)

a - stratification thermique,

b - rugosité avec l'hypothèse $z_{o h}=z_{o}$,

c - rugosité avec résistance supplémentaire. 


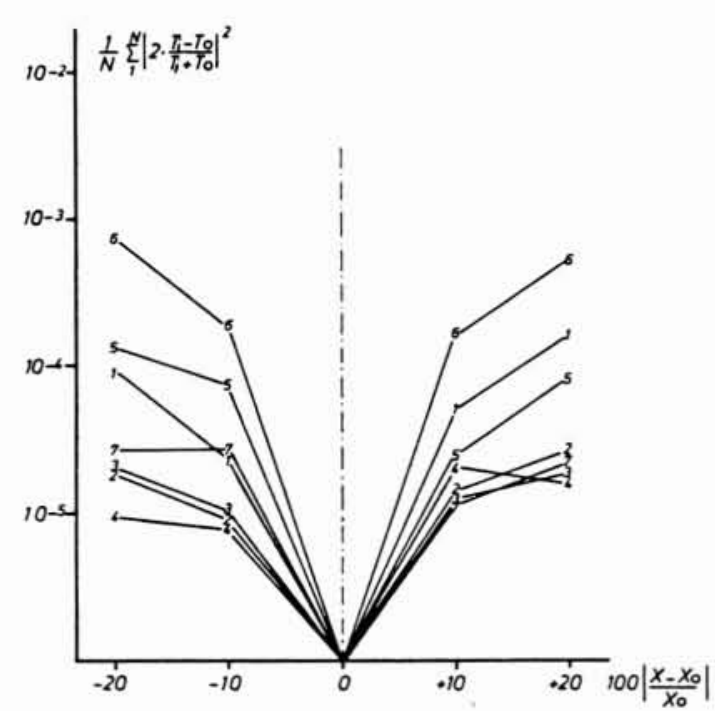

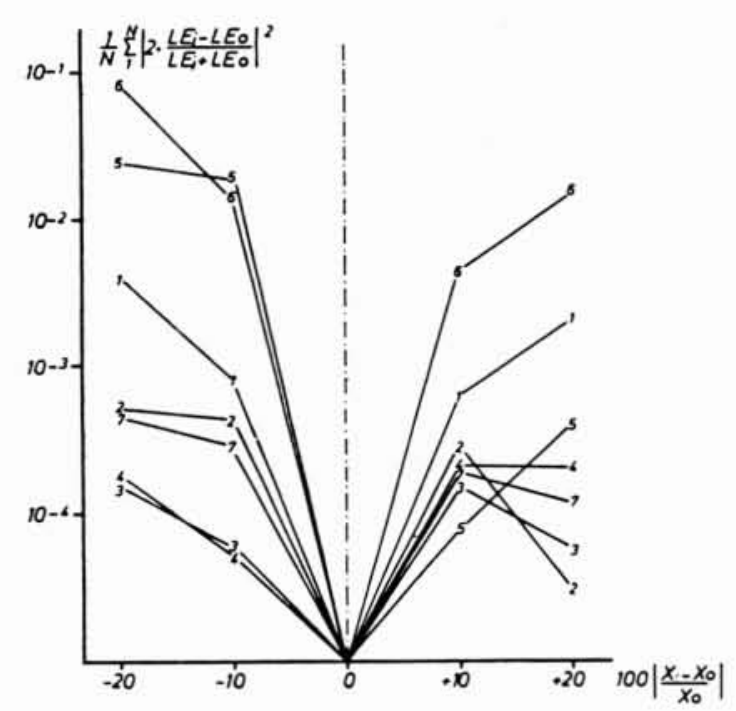

b
- les caractéristiques thermiques et hydriques du sol (conductivités $D_{m h}$ et $D_{c T}$, relation pression-humidité $h(\eta)$ ) sont d'un grand poids. Une étude de sensibilité (fig. 4, d'après BRUNET, 1984) montre leur influence respective sur quatre variables de sortie (profils de température et d'humidité du sol, flux de chaleur latente et de conduction) dans le cas d'un sol stratifié. La figure 5 illustre l'influence de la conductivité hydraulique à saturation $K_{s}$ (telle que $K / K_{s}=f\left(\eta / \eta_{s}\right)$ ) sur le flux de chaleur latente à la surface du sol : une erreur de $100 \%$ sur $K_{s}$ (ordre de grandeur réaliste étant donnée l'imprécision de la mesure et sa variabilié spatiale) entraîne ici une variation du flux de chaleur de plus de $200 \mathrm{Wm}^{-2}$, ce qui, pour ces conditions, correspond à une variation de température de surface de plus de $10^{\circ} \mathrm{C}$.

Cet exemple justifie à lui seul la nécessité d'étudier le problème de la variabilité spatiale des propriétés du sol, ce que nous allons maintenant envisager.

\subsection{Variabilité spatiale des caractéristiques hydrodynamiques}

Si très peu de connaissances sont à l'heure actuelle acquises sur la variabilité spatiale des propriétés thermiques des sols, de nombreuses études ont montré depuis une vingtaine d'années qu'à l'échelle d'une même parcelle les caractéristiques hydrodynamiques (relations $h(\eta)$ et $K(\eta))$ pouvaient varier d'un ou plusieurs ordres de grandeur (Nielsenet al.,1973; VAUCLIN et al., 1981). A l'aspect déterministe de la modélisation vient donc s'ajouter la nature stochastique de ces paramètres, qui complique a priori lourdement le problème.

La théorie de similitude en milieux poreux (Miller et Miller, 1958) permet fort heureusement de le résoudre de manière relativement simple. Elle consiste à abandonner l'hypothèse d'homogénéité pour celle d'hétérogénéité d'échelle : deux milieux différents (par exemple deux sites sur une parcelle) vont être supposés en similitude géométrique, et ne vont donc se distinguer que par une longueur interne caractéristique (fig. 6). On montre par analyse dimensionnelle que dans ces conditions les courbes $h(\eta)$ et $K(\eta)$ sont homothétiques entre elles; pour une même teneur en eau, on peut écrire :

$$
h_{i}=\alpha_{i}^{-1} h_{m} ; K_{i}=\alpha_{i}^{2} K_{m}
$$

(l'indice $i$ se refère à un milieu quelconque et $m$ à un milieu de référence; est le "facteur d'échelle" pour le milieu $i\left(\alpha_{i}=l_{i} / l_{m}\right)$. Cette théorie semble, dans les conditions naturelles, rendre compte pour une large part de la dispersion spatiale des caractéristiques hydrodynamiques (WARRICK et al., 1977; SIMMONS et al., 1979), et le facteur d'échelle présente le plus souvent une distribution log-normale.

Du point de vue de la modélisation, ces résultats sont très séduisants : on va ainsi pouvoir appréhender l'ensemble de la variabilité spatiale des propriétés hydrodynamiques d'un sol donné à l'aide d'un unique paramètre, de nature typiquement stochastique, le facteur d'échelle.

Une étude de l'influence de ce paramètre sur les variables de sortie du modèle a été entreprise (BRUNET, 1984). Son principe est le suivant :

- on définit des conditions de simulation de base : 2 jours de pluie suivis de 8 jours d'assèchement, avec les variables climatiques présentées en figure 7;

- on considère un sol donné (limon argileux des Vignères, Vaucluse) présentant deux strates différentes, auquel on attribue une distribution de facteur d'échelle définie par :

$$
m_{\log a}=-0.616 ; \sigma_{\log a}=1.16
$$

- on réalise $N$ simulations $(N=50$ ), avec autant de valeurs de $\alpha$ prises conformément à sa loi de distribution; - on étudie les résultats en terme de valeur moyennes et de dispersion autour de ces valeurs. 

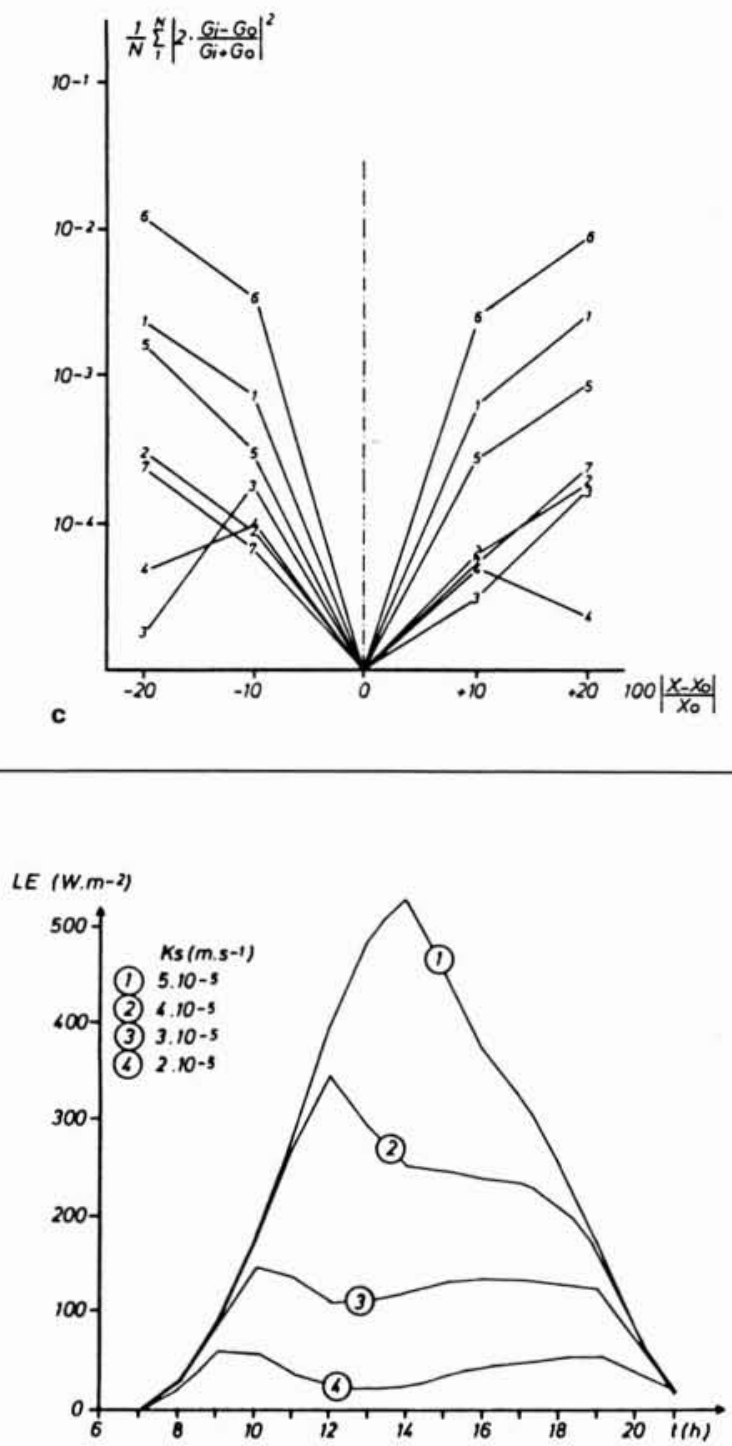

Figure 5. - Influence de la conductivité hydraulique à saturation sur le flux de chaleur latente.

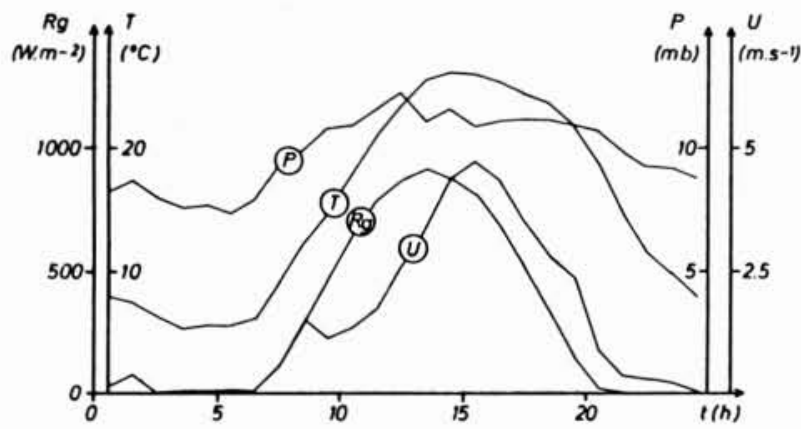

Figure 7. - Caractéristiques climatiques utilisées pour les simulations présentées figure 8,9 .

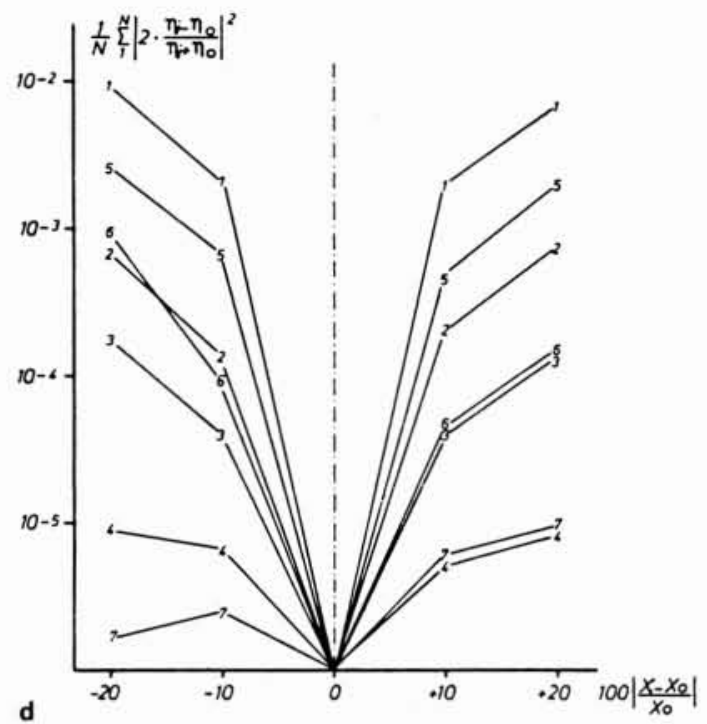

Figure 4. - Analyse de sensibilité à différents paramètres du modèle dans le cas d'un sol stratifié (d'après Brunet, 1984).

1 - pression de référence - couche 1 ,

2 - conductivité hydraulique à saturation - couche 1 ,

3 - pression de référence - couche 2 ,

4 - conductivité hydraulique à saturation - couche 2 ,

5 - facteur d'échelle,

6 - coefficient d'affinité pour la conductivité thermique.

7 - longueur de rugosité aérodynamique.
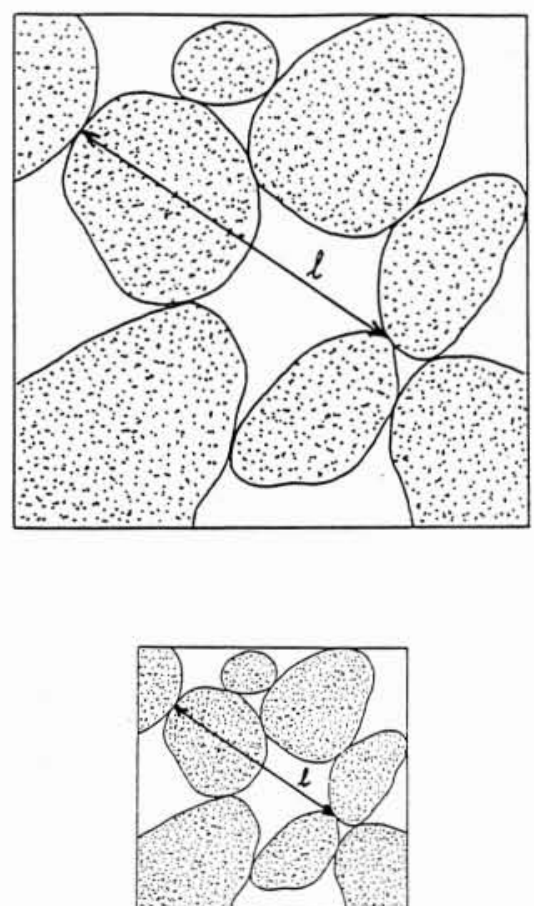

Figure 6. - Similitude géométrique en milieux poreux (1 : longueur interne caractéristique). 


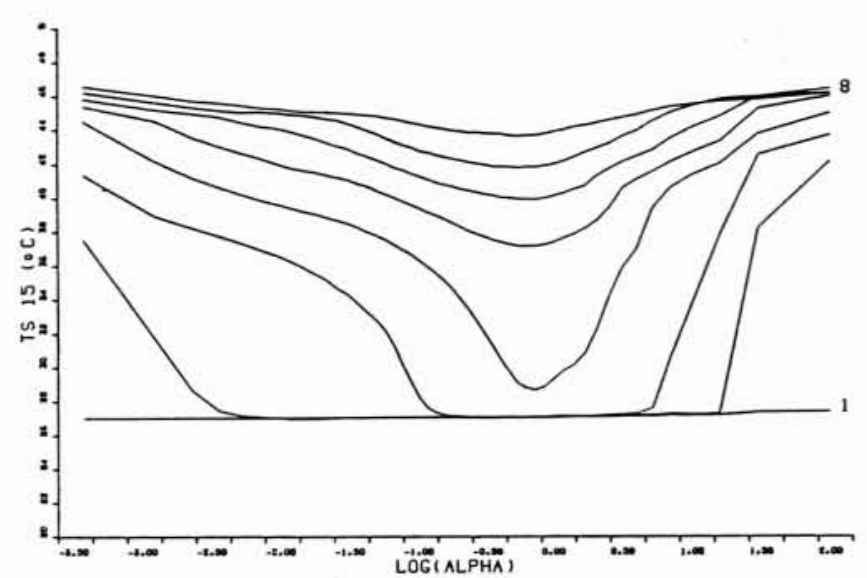

Figure 8. - Température de surface du sol calculée à 15 heures, en fonction du facteur d'échelle (d'après Brunet, 1984).

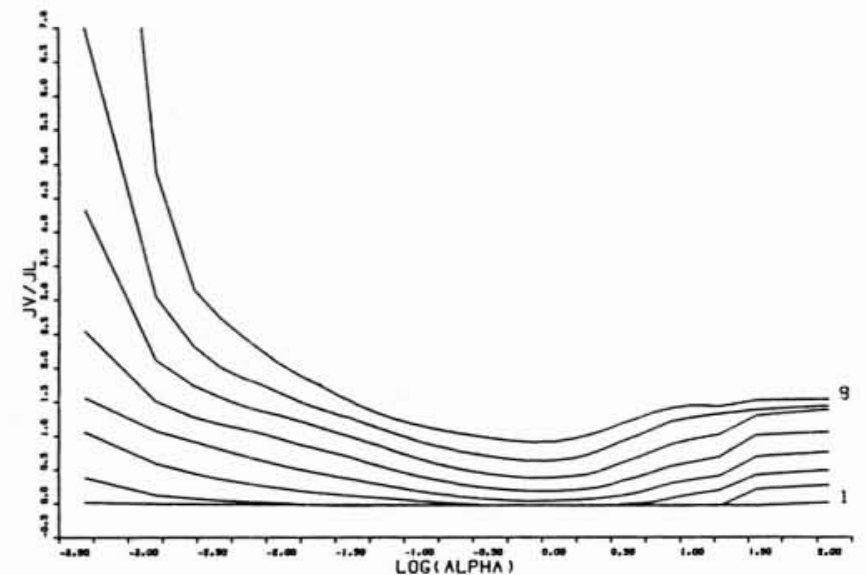

Figure 9. - Rapport des flux journaliers vapeur et liquide en fonction du facteur d'échelle (d'après Brunet, 1984).
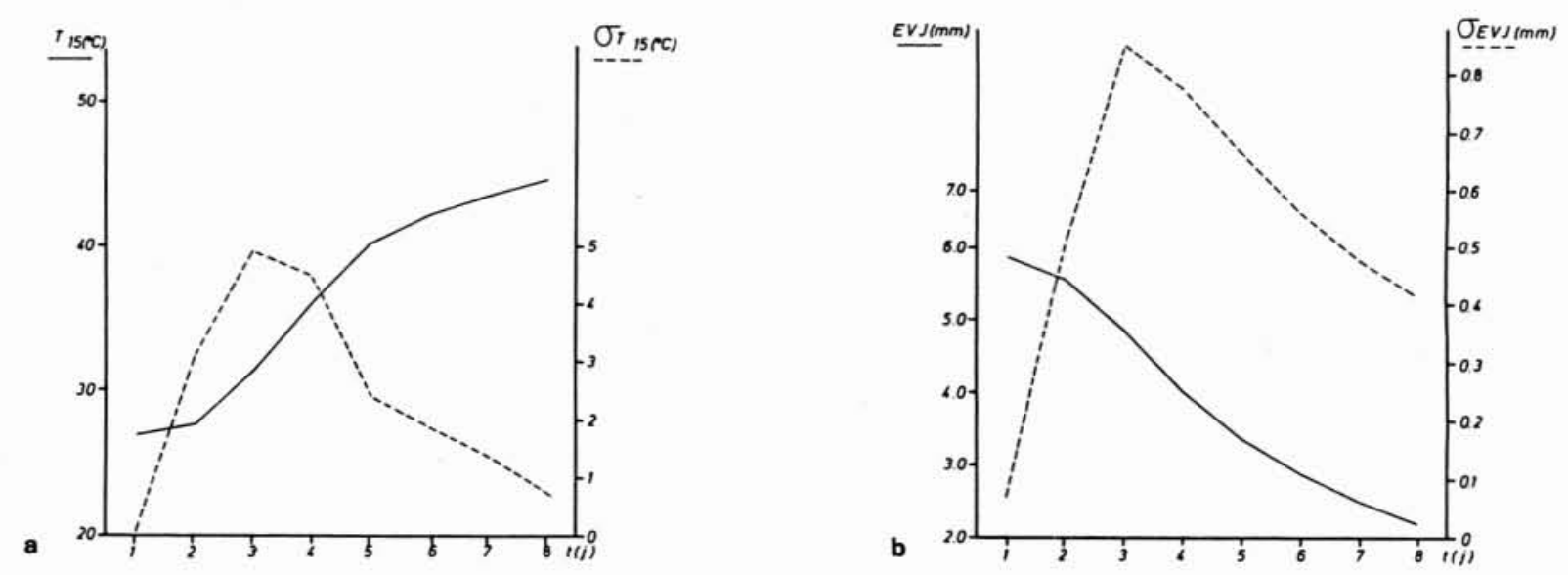

Figure 10. - Evolution des moyennes et des écarts types (d'après Brunet, 1984).
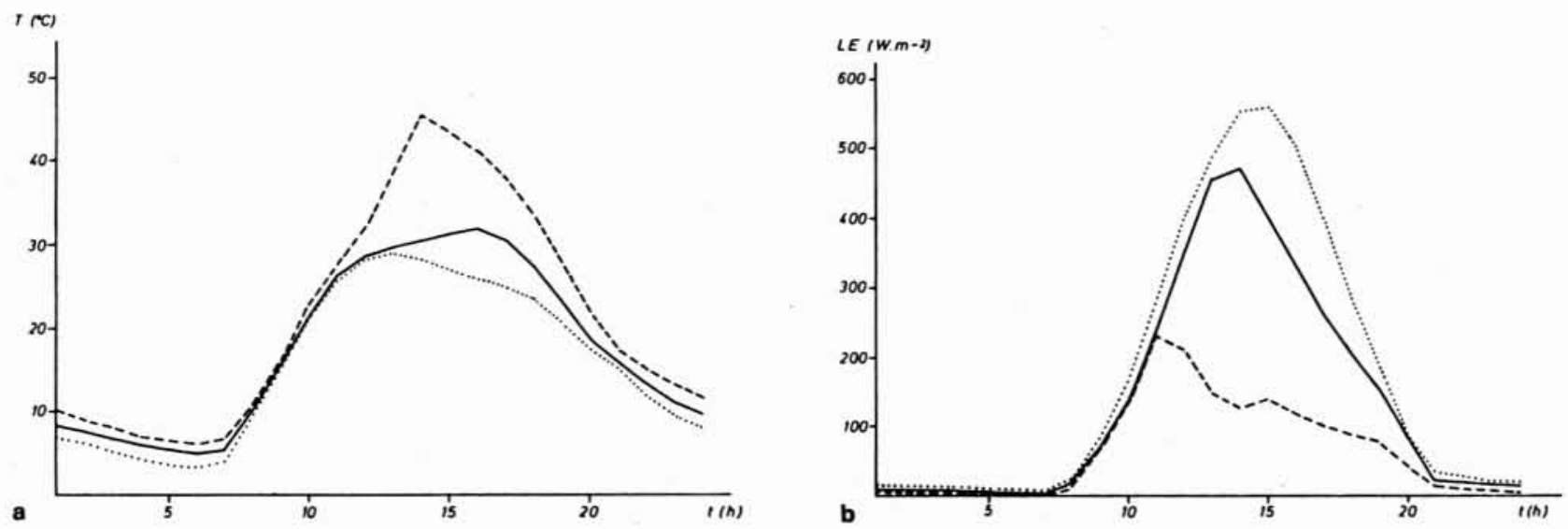

Figure 11. - Exemples d'évolutions horaires de la température de surface et du flux de chaleur latente (d'après Brunet, 1984). $(---)$ : Valeurs moyennes, $(-)$ : Valeurs minimales, (....) : Valeurs maximales. 

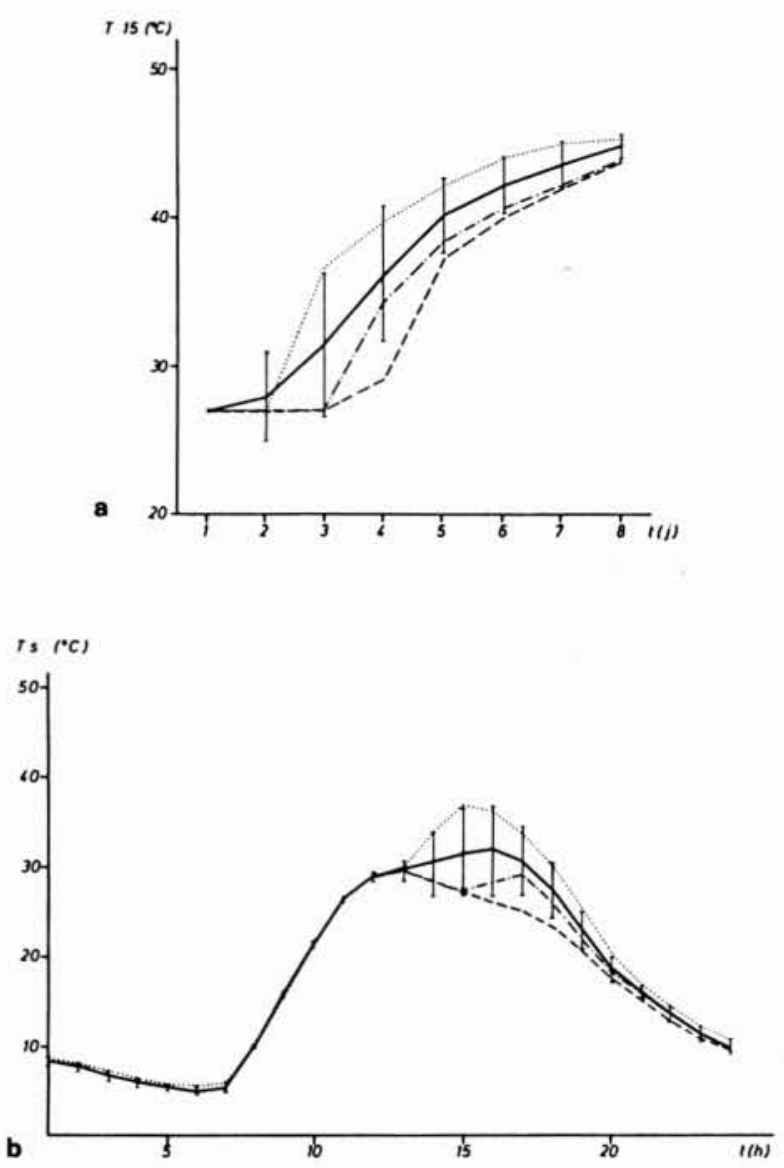

Figure 12. - Solutions stochastiques (-- $)$ et déterministes : (.....) : Modale,

(-): Moyenne,

$(-.-)$ : Médiane, (d’après Brunet, 1984).
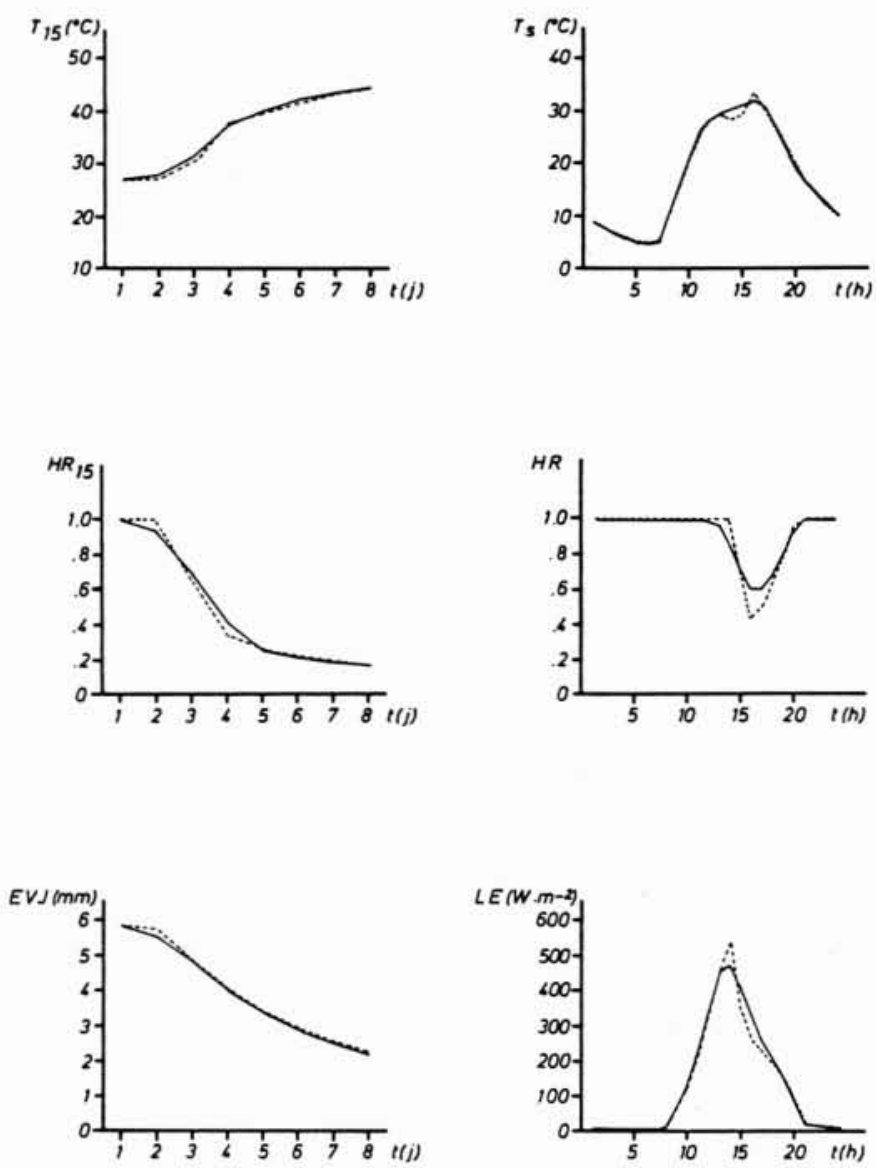

Figure 13. - Comparaison entre moyennes des simulations $(-)$ et simulations déterministes avec $\alpha=0.368$ (-), (d'après Brunet, 1984).
Les principaux résultats peuvent se résumer de la manière suivante :

- les sols à faible ou fort facteur d'échelle (peu ou fortement conducteurs) se comportent de manière semblable (fig. 8).

- en début de simulation (sols très humides; l'évaporation ne dépend que des conditions climatiques, elle est dite " potentielle ") et en fin (sols secs en surface; les transferts en phase vapeur sont importants: voir figure 9), la dispersion des résultats est faible (fig. 10);

- la variabilité "explose" lorsque les premiers sites quittent le régime d'évaporation potentielle; la variance passe par un maximum et l'on peut alors enregistrer des écarts de température de l'ordre de $20^{\circ} \mathrm{C}$, témoins de variations d'évaporation du simple au quadruple (fig. 11).

Si donc cette approche permet de simuler le comportement moyen du sol, son coût en temps-calcul rend rédhibitoire son utilisation en routine à des échelles de temps compatibles avec les problèmes de stockage/déstockage de chaleur dans le sous-sol. On peut alors envisager une alternative : n'est-il pas possible de restituer le comportement moyen du sol à l'aide d'une valeur unique du facteur d'échelle, utilisée de manière déterministe ? Un essai avec des valeurs caractéristiques de la distribution (mode, moyenne, médiane de $\log \alpha$ ) s'avère décevant (fig. 12). Par contre nous avons pu mettre en évidence que la valeur de $\alpha$ qui minimise l'écart entre solution déterministe et solution stochastique restitue convenablement ce comportement moyen (fig. 13).

$\mathrm{Ce}$ résultat encourageant ne doit pas masquer le fait qu'un " étalonnage " préalable de ce facteur d'échelle est indispensable - donc de nombreuses mesures - puisqu'il n'est pas a priori une valeur caractéristique de sa distribution. Si la méthode s'avère trop lourde, il faut alors chercher d'autres moyens de traiter les transferts.

\subsection{Une paramétrisation des transferts dans le sol}

Ce souci de simplification conduit à se tourner vers des modèles "paramétriques "; par "paramétrisation ", on entendra ici : "prise en compte des transferts à une échelle donnée, par une loi permettant de contourner les difficultés liées à la formalisation directe du phénomènes ". 

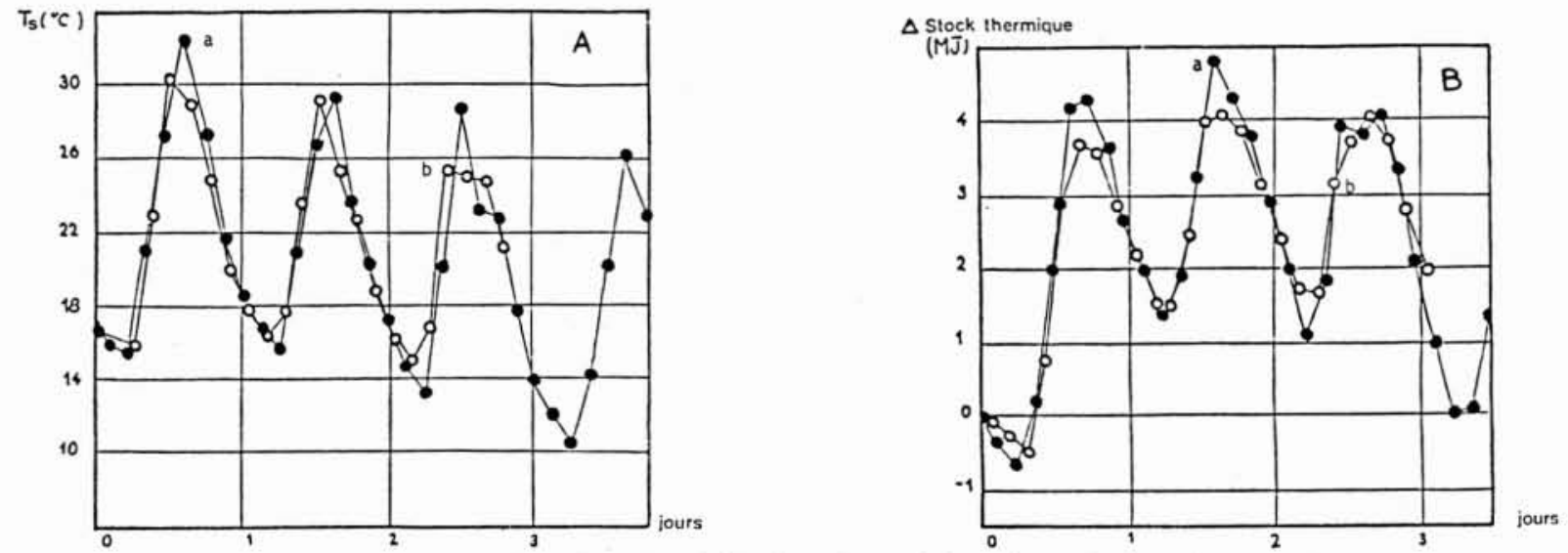

Figure 14. - Evolution de la température de surface du sol (14a) et des variations du stock thermique sur $10 \mathrm{~m}$ de profondeur (14 b), (d'après Dunand et al., 1980).

- a - modélisation globale, o b - modélisation simplifiée (Eqs 11 et 12).

Parmi ces modèles, celui dit de la «force-restore method " semble actuellement le plus pertinent. Etabli par BHUMRALKAR (1975) et BLACKADAR (1976) pour les transferts thermiques, son principe est le suivant : sous l'hypothèse d'une variation sinusoïdale de la température de surface, une combinaison de la solution analytique de l'équation de la chaleur et du bilan d'énergie à la surface conduit à :

$$
\begin{array}{r}
\frac{\partial T_{s}}{\partial t}=-C_{1}\left(\frac{-R n+H+H+L E}{\rho c_{p} d}\right) \\
-C_{2}\left(\frac{T_{s}-T_{b}}{\tau}\right)
\end{array}
$$

Le premier terme de droite permet à la surface du sol de répondre aux sollicitations atmosphériques tandis que le second tend à rétablir exponentiellement $T_{s}$ vers la température moyenne $T_{b}$ de l'épaisseur de sol considérée; $d$ et $\tau$ sont, respectivement, une profondeur d'amortissement et une constante de temps, et $C_{1}$ et $C_{2}$ deux constantes adimensionnelles dépendant des caractéristiques du matériau et de la pulsation d'attaque.

La paramétrication a ensuite été étendue aux transferts hydriques par DEARDORFF (1977), qui pose :

$$
\frac{\partial \eta_{s}}{\partial t}=-C_{1}^{\prime}\left(\frac{E-P}{\rho_{l} d^{\prime}}\right)-C_{2}^{\prime}\left(\frac{\eta_{s}-\eta_{t}}{\tau^{\prime}}\right)
$$

avec des notations équivalentes.

Cette approche a été testée pour les transferts thermiques (DEARDORFF, 1978; DUNAND et al., 1980 : voir figure 14 ) et les transferts hydriques (DEARDOFF, 1977; VAUCLIN, VACHAUD, 1981). Elle semble, au vu de ces premiers résultats, donner satisfaction. Ce n'est pas le cas en présence d'un système de stockage de chaleur très proche de la surface : une rapide étude de sensibilité montre que sa profondeur doit être supérieure à $0.6 \mathrm{~m}$. (DUNAND et al., 1980).

Intéressante par les pas de temps qu'elle permet d'adopter ( $3 \mathrm{~h}$. par exemple pour les résultats consignés en fig. 14), la méthode est en outre beaucoup moins exigeante au niveau des paramètres d'entrée que le modèle complet. Cependant, aucune étude n'a été entreprise sur sa sensibilité aux coefficients adimensionnels, ni à leurs variations éventuelles avec les types de sol ou de conditions climatiques; d'autre part, les deux équations précédentes n'ont à notre connaissance jamais été résolues et testées simultanément, ce qui est pourtant indispensable si l'on veut simuler de longues périodes de stockage ou de déstockage de chaleur.

\section{Conclusion}

Le choix d'un modèle thermique est avant tout fonction des objectifs poursuivis (épaisseur de sol concernée, extension horizontale, longueur de la période de simulation...) et des moyens disponibles pour les satisfaire (nature des mesures et quantité, outil informatique...), qui conditionnent la précision finale.

S'il est clair que dans nombre de cas, un modèle purement conductif peut s'avérer trop grossier, d'autres possibilités sont envisageables, du modèle mécaniste complet aux modèles de type paramétriques. Dans l'état actuel des connaissances, leur emploi n'est cependant pas immédiat puisqu'on ne sait pas relier leurs paramètres constitutifs à des caractéristiques simples du système physique considéré (complexe sol-atmosphère), à supposer que cela soit possible.

L'effort doit être poursuivi dans cette voie, et les deux approches (mécaniste / paramétrique) peuvent être complémentaires : une fois correctement validé un modèle du premier type, des expériences numériques poussées doivent permettre d'établir des lois paramétriques plus simples. 


\section{Références bibliographiques}

ANDERSON D.M., A. LiNVILLE (1962). - Température fluctuations at a wetting front : I. Characteristic temperature-time curves. Soil Sci. Am. Proc., 26, 14-18.

BHUMRALKAR C. (1975). - Numerical experiments on the computation of ground surface temperature in an atmospheric general circulation model. J. Appl. Meteor., 14, 1246-1258.

BLACKADAR A.K. (1976). - Modeling the nocturnal boundary layer. Preprints third symp. atmospheric turbulence, diffusion and air quality, Raleigh, Amer. Meteor. Soc., 46-49.

BRUNET Y. (1982). - Les transferts de masse et d'énergie entre sol et atmosphère: mise au point d'un modèle numérique local. Mémoire de DEA de Mécanique Expérimentale des Fluides et Transferts Thermiques, Université de paris VI, $110 \mathrm{pp}$.

BRUNET Y. (1984). - Modélisation des échanges sol nu-atmosphère; essai de validation locale et influence de la variabilité spatiale du sol. Thèse de Docteur-Ingénieur, USMG-INPG, Grenoble, 201 pp.

BRUTSAERT W. (1975). - On a derivable formula for long wave radiation from clear skies. Water Resour. Res., 11, 742-744.

BRUTSAERT W. 1982. Evaporation into the atmosphere. Reidel Ed., $299 \mathrm{pp}$.

Crausse P., G. Bacon, S. Bories (1981). - Étude fondamentale des transferts couplés chaleur-masse en milieu poreux. Int. J. Heat, and Mass Transfer, 24, 991-1004.

DEARDORFF J.W. (1977). - A parametérization of ground surface moisture content for use in atmospheric prediction models. $J$. Appl. Meteor., 16, 1182-1185.

DEARDORFF J.W. (1978). - Efficient prediction of ground surface temperature and moisture, with inclusion of a layer of vegetation. J. Geophys. Res., 83, 1889-1903.

Dunand A., M. Vauclin, G. Vachaud (1980). - Simulation du comportement thermique du sol autour d'un échangeur enterré de pompe à chaleur. $T$ symposium international de l'A.I.R.M., "Transferts de chaleur et de masse en milieu poreux ".

Groenevelt P.M., B.D. KaY (1974). - On the interaction of water and heat transport in frozen and unfrozen soils : (II). The liquid phase. Soil Sci. Soc. Amer. Proc., 38, 400-404.
Miller E.E., R.D. Miller (1956). - Physical theory for capillary flow phenomena. J. Appl. Phys., 27, 324-332.

MILLY P.C.D. (1982). - Moisture and heat transport in hysteretic, inhomogeous porous media : a matric head-based formulation and numerical model. Water Resour. Res., 18, 489-498.

Nielsen D.R., J.W. Biggar, K.T. ERH (1973). - Spatial variability of field-measured soil-water properties. Hilgardia, 42, 215-259.

RECAN M. (1982). - Modélisation mathématique du comportement thermique d'un sol nu; application à la télédétection dans l'infra-rouge. Thèse de Docteur-Ingénieur, Institut National Polytechnique de Toulouse, $98 \mathrm{pp}$.

Rosema A. (1975). - Simulation of the thermal behavior of bare soils for remote sensing purposes. In "Heat and mass transfers in the biosphere ": I. Ed. DE VRIES D.A., AfGan H.H., 109-135.

Simmons C.S., D.R. Nielsen, J.W. Biggar (1979). - Scaling of field measured soil water properties. Hilgardia, 47, 77-174.

VAN BAVEl C.H.M., D.I. Hillel (1976). - Calculating potential and actual evaporation from a bare soil surface by simulation of concurrent flow of water and heat. Agric. Meteor., 17, 453-476.

Vauclin M., G. Hamon, G. Vachaud (1977). - Simulation of coupled flow of heat and water in a partially saturated soil. Determination of the surface temperature and evaporation rate from a bare soil. in Rapport final de l'ATP 1652 " Hy. drogéologie " CNRS "Influence des gradients thermiques sur les transferts d'humidité dans la zone non saturée ", I.34-I.52.

Vauclin M., Haverkampx, G. Vachaud (1979). - Résolution numérique d'une équation de diffusion non linéaire. Application à l'infiltration de l'eau dans les sols non saturés. Presses Universitaires de Grenoble, $183 \mathrm{pp}$.

VAUCLIN M., G. VACHAUD (1981). - Bilan hydrique dans le Sud-Tunisien. II. Modélisation numérique et pévision des transferts hydriques en sol stratifié. J. Hydrol., 49, 53-73.

VAuclin M., J. Imbernon, G. Vachaud (1981). - Spatial variability of some physical properties over one-hectare field plot. AGU Chapman Conf. Fort-Collins (Colorado), juillet.

Warrick A.W., G.J. Mullen, D.R. Nielsen (1977). - Scaling field measured soil hydraulic properties using a similar media concept. Water Resour. Res., 13, 355-362. 
L'ensemble des discussions qui se sont développées à la suite des divers exposés de cette session fait apparaître globalement deux types de préoccupations de la part des intervenants : - la connaissance précise des paramètres conditionnant les phénomènes de transfert de chaleur et d'humidité dans le sol et en surface,

- l'aptitude des modèles théoriques présentés à prévoir la réalité physique.

Monsieur GOLDSTEIN pose en premier lieu le problème de la détermination des coefficients de diffusion pris en compte au cours de simulations numériques du comportement thermique et hydrique de sols, présentées dans le cadre d'études développées à l'Institut de mécanique des fluides de Toulouse. Monsieur BORIES précise qu'en ce qui concerne la conductivité thermique, ce paramètre a été déterminé à l'aide d'une méthode expérimentale de régimes stationnaires. Les coefficients de diffusion de masse ont été, également, évalués par des expériences spécifiques, en admettant un découplage au niveau de la quantité de mouvement entre les écoulements en phase gazeuse et en phase liquide.

La présentation de relevés de températures dans un bassin sédimentaire effectuée ensuite par Monsieur WATREMEZ, soulève, entre autres, le problème de la précision des mesures in situ par forage : risque de convection autour du point de mesure (Monsieur BORIES), perturbation de la mesure par le forage lui-même (Monsieur BANAL). D'autre part, le champ de température dans le sol peut être également influencé par l'existence d'incidents de structure (batholites) d'après Monsieur GRJEBINE, ou par la présence dans les fractures, de régimes de convection naturelle (Monsieur WATREMEZ) induisant des inversions de gradients de température, soulignées par Monsieur BORIES. Les variations du paramètre conductivité thermique (de 1 à 5) utilisé dans les modèles thermiques du Bassin Parisien, paraissent élevées à Monsieur FIRDAOUSS. Monsieur WATREMEZ fait alors remarquer que ces variations, obtenues par des mesures effectuées à faible profondeur ou en laboratoire, sont normales, en raison de la présence de fluides dans les roches qui modifient sensiblement la conductivité thermique équivalente.

La représentativité des modèles théoriques utilisés et des hypothèses formulées, en vue de simuler les champs de température et d'humidité dans les sols, apparaît ensuite comme le thème principal des discussions.

En ce sens, le concept de similitude, introduit dans la modélisation de la variabilité spatiale de paramètres macroscopiques (perméabilité, conductivité, etc.) par l'exposé de Monsieur BRUNET, soulève une question de Monsieur QUINTARD quant à la justification de pratiquer la similitude sur des grandeurs macroscopiques faisant elles-mêmes intervenir des couplages de phénomènes microscopiques ayant des similitudes différentes. Monsieur BRUNET reconnaît que si cette approche implique de fortes hypothèses, elle fournit en général une bonne adéquation entre modèles et mesures, dans le cas de milieux poreux à granulométrie peu étendue (exemple des sols de type "sable "); par contre, lorsque les tailles de particules sont très différentes, la similitude n'est plus, effectivement, aussi bonne. Monsieur GRJEBINE pose le problème important du rôle joué par la couverture biologique dans la modulation des phénomènes de transfert sol-atmosphère, particulièrement lors de l'existence de mécanismes de surface séquentiels, tels que effets de pluie et évaporation de l'humidité, se traduisant par des apports ou des pertes de calories. Monsieur BRUNET souligne que, dans le cas de sols nus, des gradients thermiques très importants peuvent exister dans les couches superficielles à l'échelle de la journée, alors qu'en présence d'un couvert végétal, ils seront en moyenne beaucoup plus modérés. A l'échelle de l'année, la différence est beaucoup moins sensible au niveau du stockage de chaleur, les températures évoluant de manière semblable en profondeur. Il semble donc, comme le fait remarquer Monsieur le Président, que la prise en compte de phénomènes de type biologique dans les modèles prévisionnels de transfert permettrait une approche plus précise de la réalité, en particulier lors des opérations (sensiblement différentes) de séchage de grains de nature biologique ou minérale.

Monsieur BENET, après avoir décrit une modélisation mathématique locale des sols non saturés, basée sur la Thermodynamique des Processus Irréversibles, présente des résulats expérimentaux sur le comportement thermique et hydrique de la couche d'un sol située au-dessus d'un stockage de chaleur. Monsieur GRJEBINE s'interroge à nouveau quant à l'incidence sur ces résultats de la présence en surface d'une couche imperméable à la vapeur. Monsieur BENET répond que dans la configuration expérimentale étudiée, les transferts d'énergie en phase gazeuse, et également en phase liquide sont négligeables. Monsieur BORIES fait alors remarquer qu'après les exposés de MM. BRUNET et BENET on peut finalement s'interroger quant à l'intérêt d'avoir recours à des modèles compliqués, quand on se rend compte que dans les deux cas, on arrive à rendre compte de certaines expériences avec des simplifications draconniennes. Il précise qu'en l'occurence les deux cas présentés ne sauraient avoir valeur de règle générale et qu'il convient de procéder à des analyses de sensibilité aux paramètres avant toute simplification a priori. Des travaux effectués à Toulouse par MM. RECAN et CRAUSSE illustrent d'ailleurs parfaitement les risques auxquels peuvent conduire ces réductions de modèles dans l'étude des transferts couplés en milieu poreux. Monsieur BRUNET souligne qu'on ne peut parler d'un modèle qu'en fonction des objectifs qu'on lui assigne. Monsieur BENET précise alors qu'il faut quand même distinguer les modèles permettant une analyse fine de la physique des phénomènes et des modèles de prévision liés à une application.

Monsieur le Président conclut en développant l'idée que le modèle général est élaboré pour aborder toute situation, puis on le simplifie, compte tenu des variables qui paraissent essentielles dans la configuration physique étudiée.

Nota: Le texte ci-dessus fait la synthèse des discussions qui ont suivi la présentation des quatre communications présentées le 14 novembre 1984 sur "les transferts thermiques dans le sous-sol ".

Cette synthèse a été préparée par M. P. Crausse, Maitre de Conférences à l'INP de Toulouse.

Pour la commodité des lecteurs, nous la présentons à la suite de chacune des quatre communications concernées. 